\title{
Metastatic Bladder Cancer: A Review of Current Management
}

\author{
Andrew Fletcher, ${ }^{1}$ Ananya Choudhury, ${ }^{2,3}$ and Nooreen Alam ${ }^{2}$ \\ ${ }^{1}$ Department of Palliative and Supportive Care, The Christie NHS Foundation Trust, Wilmslow Road, Manchester M20 4BX, UK \\ ${ }^{2}$ Department of Clinical Oncology, The Christie NHS Foundation Trust, Wilmslow Road, Manchester M20 4BX, UK \\ ${ }^{3}$ The University of Manchester, Oxford Road, Manchester, M13 9PL, UK \\ Correspondence should be addressed to Ananya Choudhury, ananya.choudhury@christie.nhs.uk
}

Received 16 February 2011; Accepted 6 April 2011

Academic Editor: A. M. El-Assmy

Copyright ( $\odot 2011$ Andrew Fletcher et al. This is an open access article distributed under the Creative Commons Attribution License, which permits unrestricted use, distribution, and reproduction in any medium, provided the original work is properly cited.

Bladder cancer continues to result in substantial morbidity and mortality for affected individuals. Advances in the management of metastatic bladder cancer have been limited. Chemotherapy with platinum-based regimes remains the mainstay of first-line treatment. Studies investigating alternative regimes have offered no survival advantage. Targeted therapies may offer benefit either as single agent or in combination with chemotherapy. Symptoms due to metastatic bladder cancer impact patients' quality of life, and therefore holistic management is vital. Such management includes radiotherapy, bisphosphonates, and the involvement of specialist palliative care services. This review will discuss the current management for metastatic bladder cancer, future potential treatment modalities, and the evidence to support the management strategies.

\section{Introduction}

There were over 10,000 new cases of bladder cancer in the UK in 2008 making it the 7th commonest cancer overall. A quarter of these are muscle-invasive bladder cancers (MIBC) [1-5]. Approximately 5\% of patients present with metastatic bladder cancer at diagnosis [5]. In the UK, the most common histological type of bladder cancer is transitional cell carcinoma (TCC), however, there are other types, including squamous cell carcinoma, adenocarcinoma, and less commonly small cell and small cell and sarcoma. Factors such as geography and underlying aetiology influence prevalence across the world [6]. This review will discuss the current management of patients with metastatic bladder cancer, in the main of the TCC type, and the evidence to support this management.

\section{Chemotherapy}

2.1. First-Line Chemotherapy. Combination chemotherapy is the treatment of choice for metastatic bladder cancer. Methotrexate, vinblastine, doxorubicin, and cisplatin (MVAC) was for many years the preferred regime; however, patients experienced high toxicity levels. Newer chemotherapy regimes have attempted to offer comparable or better efficacy in terms of overall survival, response rates, and time to disease progression whilst decreasing toxicity (Table 1).

In an attempt to offer the benefits of MVAC whilst reducing toxicity, especially neutropenic sepsis, Sternberg et al. [7] undertook a multicentre study of 263 patients with metastatic or advanced urothelial tumours who had not previously received systemic chemotherapy and randomised them to receive traditional MVAC or high-dose-intensity MVAC plus granulocyte colony-stimulating factor (HDMVAC + GCSF). HD-MVAC + GCSF when compared with MVAC offered statistically lower levels of grade 3 and 4 neutropenia (20\% versus $62 \%, P<.001)$. Although median survival was unaffected, there was a statistically significant improvement in complete response $(25 \%$ versus $11 \%, P=$ $.006)$ and overall response rate (70\% versus $58 \%, P=.016)$.

Gemcitabine and cisplatin (GC) been compared with MVAC in a phase-III randomised controlled trial, which showed that GC had significantly less toxicity with significantly lower rates of neutropenic sepsis and grade 3 or 4 mucositis and a reduction in drug-related mortality, though the latter was not statistically significant [8]. Response rates 


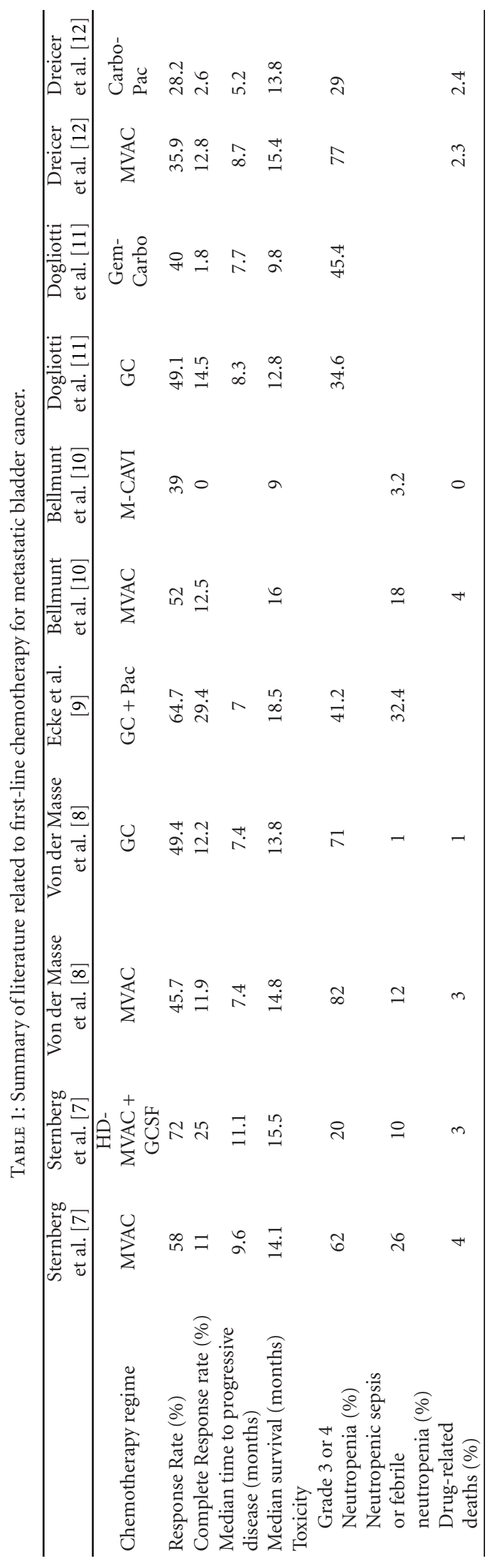


for GC versus MVAC were $49.4 \%$ and $45.7 \%$, respectively, median survival of 13.8 and 14.8 months, and the time to progressive disease was identical in both groups at 7.4 months. Furthermore, there were statistically significant higher number of patients with a greater than $5 \%$ increase in weight in the GC group and a reduction in fatigue, although this was not significant. Other quality-of-life markers were maintained and were similar in both arms.

Triplet combinations with GC have also been studied, including a single-centre study of the triplet combination of GC plus paclitaxel [9]. Median survival and median progression-free survival were 18.5 months and 7 months, respectively, with a reported response rate of $64.7 \%$. Neutropenia was experienced in $41.2 \%$ of participants and neutropenic sepsis in 32.4\%.

Platinum-containing chemotherapy is the gold standard for patients with metastatic bladder cancer, however, some patients have inadequate renal function or do not tolerate cisplatin, for example due to neuropathy. In these patients, carboplatin has been suggested as an alternative. Bellmunt et al. [10], Dogliotti et al. [11], and Dreicer et al. [12] compared cisplatin with carboplatin-containing regimes. Bellmunt et al. [10] randomised 47 patients to MVAC or methotrexate, carboplatin, and vinblastine (M-CAVI). There was a statistically higher median survival in the MVAC group (16 months versus 9 months, $P=.03$ ); however, response rates were similar. Gemcitabine plus carboplatin was compared with GC in a study of 110 chemonaive patients by Dogliotti et al. [11]. No statistically significant differences were demonstrated in terms of response rate $(40 \%$ versus $49.1 \%)$, complete response (1.8\% versus $14.5 \%)$, median time to progression (7.7 months versus 8.3 months), median survival (9.8 months versus 12.8 months), or toxicity. Dreicer et al. [12] undertook a phase-III study comparing MVAC versus paclitaxel plus carboplatin, following observation of previous good response. In this trial, there was no statistically significant improvement in overall survival and response rates or difference in quality of life, but there were higher rates of neutropenia in those receiving MVAC.

A further study by Carles et al. [13] undertook a multicentre phase-II study using oxaliplatin and gemcitabine. Chemo-naive as well as patients who had received previous chemotherapy were permitted to enter the study. Complete response was $6.5 \%$ with an overall response rate of $48 \%$. At the end of study, most of the patients (90\%) had progressive disease with a median time to disease progression of 5 months and a median overall survival of 6.5 months. Grade 3 or 4 neutropenia was reported in $22 \%$ of cases.

2.2. Second-Line Chemotherapy. Potential second-line options in metastatic bladder cancer include single-agent vinflunine, taxanes and combination regimes (Table 2). Studies often select patients with performance status 0 and 1 for their inclusion criteria, which may not reflect the generally poorperformance status represented by patients with metastatic bladder cancer.

A phase-III multi-centre study randomised 370 patients who had previously progressed through platinum-based chemotherapy, to receive vinflunine and best supportive care versus best supportive care alone [14]. Overall survival and progression-free survival were marginally, albeit significantly longer in those receiving vinflunine, 6.9 months versus 4.3 months $(P=.04)$, and 3 months versus 1.5 months, respectively. Vinflunine did not impair quality of life.

A phase-II study recruited 45 patients who were treated with weekly paclitaxel [15]. Overall response was 9\%, complete response $2 \%$, median time to progression 3 months, and median survival 7 months. Febrile neutropenia rates were $4 \%$. Two weekly versus 3 weekly gemcitabine plus paclitaxel was investigated by Fechner et al. [16]. Median time to progression and median survival were not statistically different, however, there was a statistically significant increase in complete response rate in the 3-weekly regime, though this was a small study.

Pemetrexed with vitamin B12, folic acid, and dexamethasone prophylaxis was investigated in a phase-II study to treat 47 patients with progressive disease following adjuvant or neoadjuvant chemotherapy [17]. Complete response, partial response, and overall response were $6.4 \%, 21.3 \%$, and $27.7 \%$, respectively. Median duration of response was 5 months and median overall survival 9.6 months. Neutropenic rates were $4.3 \%$.

\section{Targeted Therapies}

3.1. Histone Deacetylase Inhibitors (HDACi). An imbalance in the equilibrium of histone acetylation has been associated with carcinogenesis and cancer progression. HDAC inhibitors (HDACis) lead to an accumulation of acetylated histone proteins in both tumour cells and in normal tissues. They activate differentiation, arrest the cell cycle in G1 and/or G2, and induce apoptosis in transformed or cancer cells. In vitro and in vivo, HDACi demonstrate antitumour activity, by leading to an accumulation of acetylated histones and cell cycle arrest and/or apoptosis of some transformed cells.

HDACi are currently being investigated in ongoing research studies both in the locally advanced and metastatic setting [23]. Early phase trials have also investigated using HDACi concurrently with radiotherapy and chemoradiotherapy [24-27].

\subsection{Epidermal Growth-Factor-Receptor-Targeted Therapy. Overexpression of epidermal growth factor receptors includ- ing human epidermal growth factor receptor (EGFR/HER) has been demonstrated in bladder cancer and may offer both prognostic indicators as well as a potential targeted treatment for metastatic bladder cancer with recommendations sug- gesting to be used as a supplement to conventional chemo- therapy.}

3.2.1. Trastuzumab. Trials are currently ongoing; however, a small study by Peyromaure et al. [28] included 6 patients with metastatic urothelial cancer and overexpression of HER2. The participants received trastuzumab and paclitaxel; carboplatin, trastuzumab, and paclitaxel, or trastuzumab alone. After 3 cycles, all patients demonstrated a partial response between 30 and $70 \%$. After 6 cycles, 5 patients 


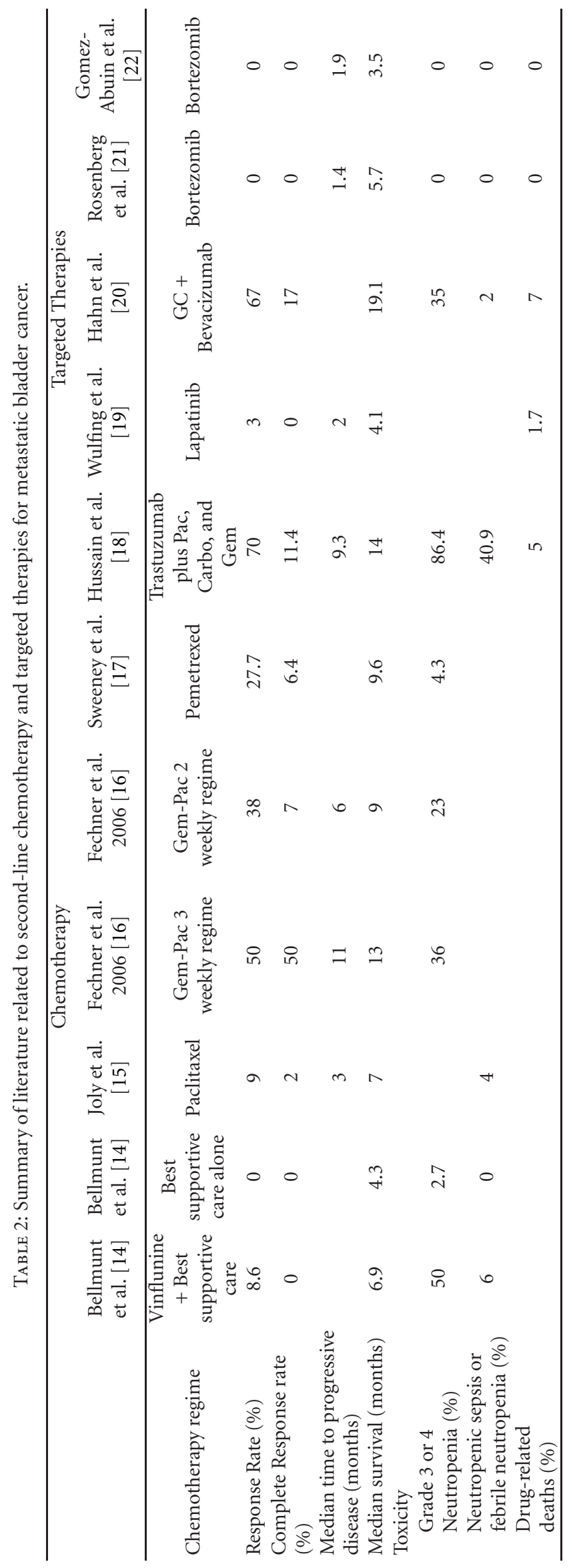


had progression of at least 1 site of metastatic disease. In the five patients who died, the interval between trastuzumab initiation and patient death ranged from 8 to 18 months. One patient who received trastuzumab alone remained alive at 28 months. Grade 4 neutropenia was documented in 1 patient, and there were no cases of congestive heart failure. The small number of patients included in the study limit the conclusions that can be drawn from the results, however, highlight the need for larger trials to investigate the effectiveness of trastuzumab therapy alone and in combination with chemotherapy.

The safety and efficacy of trastuzumab in combination with carboplatin, gemcitabine, and paclitaxel was investigated as part of a phase-II National Cancer Institute trial [18]. Patients with over expression of HER-2/neu receptor on immunohistochemistry, gene amplification, and/or elevated serum HER-2/neu, who had not received chemotherapy for metastatic disease, were treated with a regime comprising all the four drugs. In those treated, there was a $70 \%$ overall response with a complete response in $11.4 \%$. Median time to progression was 9.3 months and median survival 14 months. Toxicities included: grade 3 neutropenia (16\%), grade 4 neutropenia $(70 \%)$, infection-related deaths $(5 \%)$, overall cardiac toxicity (14\%), and grade III sensory neuropathy (14\%).

In a further phase-II study, Beuzeboc et al. [29] aimed to demonstrate a progression-free survival benefit for those treated with trastuzumab. Interim safety results concluded that there were no statistically significant differences in the rates of toxicity between those receiving trastuzumab plus chemotherapy versus chemotherapy alone. Efficacy data is awaited.

3.2.2. Lapatinib. Lapatinib has an established use in HER-2positive breast cancer [30] and is being increasingly trailed in bladder cancer. In a single-arm phase-II study using Lapatinib as the second-line treatment, there is a correlation between overexpression of eGFR/HER-2 and response to this therapy; however, the statistically significant benefit relates to the overexpression of eGFR [19].

3.3. Vascular Endothelial-Growth-Factor-Targeted Therapy. A study by Bochner et al. [31] explored the association between traditional prognostic indicators and tumour angiogenesis. Estimated probability of recurrence at 5 years for lowest microvessel counts compared with highest counts were 19\% and $68 \%$; respectively, and overall 5 year survival was $68 \%$ compared to $34 \%$. Tumour angiogenesis is an independent prognostic indicator in patients with invasive bladder TCC. Therapies that block vascular endothelial growth factor and thus the development of microvessels may offer an alternative treatment in patients with metastatic bladder cancer. Current phase-II studies investigating the use of bevacizumab have shown encouraging results; however, there are concerns with regards to toxicity. One such study which combined bevacizumab with cisplatin and gemcitabine as first-line chemotherapy identified complete response in $17 \%$ of patients with an overall response rate of $67 \%$ [20]. Although the median time to progression appeared comparable to earlier studies using chemotherapy alone, the median survival appeared to be higher at 19.1 months. There were three treatment-related deaths, and $21 \%$ of patients developed venous thromboembolism.

3.4. Proteosome Inhibitors. Inhibitors of the $26 \mathrm{~S}$ proteaosome complex have been shown to arrest tumour spread and growth and suppress angiogenesis; therefore, it is suggested that they may offer potential therapeutic approaches. Bortezomib, a proteosome inhibitor, has been investigated in two phase-II single-arm studies to treat advanced or metastatic urothelial cancers $[21,22]$. Both concluded that single-agent Bortezomib is ineffective and does not offer antitumour activity in this patient group.

\section{Radiotherapy}

Radiotherapy has several roles in the management of metastatic bladder cancer including palliation of pain secondary to bone-metastases, control of advancing pelvic pathology, and a reduction in urinary symptoms, for example haematuria. A variety of potential regimes have been investigated for the efficacy and toxicity.

An MRC BA09 study by Duchesne et al. [32] compared two hypofractionated radiotherapy schedules for local symptom control of muscle-invasive bladder cancer, $35 \mathrm{~Gy}$ in 10 fractions over two weeks versus $21 \mathrm{~Gy}$ in 3 fractions over one week. 500 patients were initially randomised with 3 -month follow-up data available in 272 patients. Overall symptom improvement, defined as improvement of at least 1 symptom by 1 grade without worsening another symptom, was $71 \%$ in those receiving 35-Gy compared with 64\% in the 21-Gy arm, though this was not statistically significant. Comparing the 35 Gy group with the $21 \mathrm{~Gy}$ group for patients with specific pretreatment symptoms, urinary frequency resolved in $43 \%$ and $42 \%$, respectively, nocturia in $51 \%$ and $35 \%$, haematuria in $58 \%$ and $61 \%$, and dysuria in $47 \%$ and $49 \%$. No difference in survival or change in WHO performance status was demonstrated between the two treatment arms. Twothirds of participants reported that quality-of-life symptom scores were either unchanged or improved three months after treatment. A smaller study by McLaran et al. [33] treated 65 elderly patients with muscle-invasive bladder cancer. Treatment involved 30 Gy in 5 fractions on a weekly basis with an additional $6 \mathrm{~Gy}$ given as the sixth fraction for participants who were fit, tolerated previous treatment, and had a field size $<1000 \mathrm{~cm}^{3}$. Complete palliation of symptoms was achieved in $51 \%$ of participants. Frequency and dysuria resolved in 24\% and haematuria resolved in $92 \%$ and frequency and dysuria resolving in $24 \%$. Twenty-eight patients experienced transient worsening of their urinary symptoms with eight requiring hospital admission due to toxicities. The median symptom-free interval was 7 months (range 0-40 months).

Other common fractionation regimes in use are $20 \mathrm{~Gy}$ in 5 fractions over one week or a single 8-10 Gy fraction. 
Several large studies investigating the use of radiotherapy for painful bone-metastases have been reported, comparing single with multiple fraction regimes. A study from the Bone Pain Trial working party [34] compared 8-Gy singlefraction radiotherapy with a multifraction regime $(20 \mathrm{~Gy}$ in 5 fractions or $30 \mathrm{~Gy}$ in 10 fractions) for the treatment of skeletal-related pain in 765 patients. There were no differences demonstrated in the time to response or to first increase in pain at any time up to 12 months. Despite retreatment being twice as common in those treated with a single fraction, the authors concluded that retreatment reflected a greater readiness to retreat after a single fraction, rather than a greater need. A further study by Steenland et al. [35] compared 8 Gy single-fraction radiotherapy with $24 \mathrm{~Gy}$ in 6 fractions in 1171 patients with metastatic cancer to bone. There were no statistical differences between the two treatment arms in terms of response, change in pain medication requirements, quality of life, or side effects. Retreatment rates were higher in those receiving a single fraction. Although level of pain was the main reason for retreatment, the authors concluded, as they did in the previous study discussed, that after a single fraction of radiotherapy, doctors were more willing to retreat. Other studies comparing single- with multifraction radiotherapy for the treatment of bone-metastases-related pain have shown comparable results $[36,37]$. A Cochrane review drew similar conclusions to the evidence discussed concluding that single-fraction and multifraction radiotherapy were equally effective in relieving pain, however, single-fraction radiotherapy conferred an increased need for retreatment and higher risk of pathological fractures [38]. It was also stated that quality-of-life and economic data is required to identify the optimal treatment regime.

\section{Bisphosphonates}

Bisphosphonates, as with other cancers, have essentially three roles: the management of hypercalcaemia of malignancy, reduction in skeletal-related events in patients with bonemetastases and the management of pain related to bonemetastases. It is widely accepted that bisphosphonates reduce the risk of skeletal complications in multiple cancers, especially metastatic disease from solid tumours [39-42] and myeloma [43], and can assist the management of pain due to bone-metastases. Although more limited, there is literature to support this theory in patients with bonemetastases from bladder cancer. A study by Zaghoul et al. [42] evaluated the effect of zoledronic acid on skeletalrelated events. Patients receiving palliative radiotherapy were randomised to placebo or zoledronic acid for 6 months. They concluded that the patients receiving zoledronic acid had a lower incidence of skeletal-related events, a prolonged time to the first skeletal-related event and an increased 1-year survival.

The recommended dose to prevent skeletal-related events in patients with bone-metastases is $4 \mathrm{mg}$ zoledronic acid (Zometa), with the dose adjusted based on baseline creatinine clearance $(\mathrm{CrCl})$ in $\mathrm{mL} /$ minute [44].

\section{Palliative Care}

All the treatment options discussed are being given with palliative intent to achieve a degree of disease control and aid symptom management. Improving Outcomes in Urological Cancers published by the National Institute of Clinical Excellence in September 2002 stated that palliative care is an integral part of the management of patients with urological cancers and should be available if needed to provide symptom control as well as social, spiritual, and psychological support [45].

This belief is supported by Brierly and O'Brien [46] who reviewed urology outpatient attendances as well as inpatient admissions and concluded that palliative care services should be closely involved in the management of patients with urological cancers and that urologists should develop the skills required to offer palliative care to their patients. $75 \%$ of patients with terminal malignancy attending outpatients had problems that would have benefited from specialist palliative care; however, the number may be higher as psychosocial aspects were not addressed during the assessments [46].

\section{Conclusions}

First-line chemotherapy in the metastatic setting is GC or HD-MVAC + GCSF. The regimes offer comparable effectiveness with reduced toxicity levels compared with the traditional MVAC regime. Carboplatin-based chemotherapy regimes offer an alternative to cisplatin-based regimes where cisplatin is contraindicated. Literature reflecting second-line chemotherapy is limited and needs further investigations. The novel targeted therapies discussed may offer additional therapeutic options either in combination with chemotherapy or as single agents. Further studies would also benefit from a greater emphasis on quality-of-life measures including pain control, which in the palliative setting, is as important as the potential response to treatment or survival advantage.

\section{Key Points}

(i) First-line chemotherapy in the metastatic setting is gemcitabine plus cisplatin or high dose intensity methotrexate, vinblastine, doxorubicin, and cisplatin (MVAC) plus granulocyte-colony-stimulating factor (GCSF).

(ii) Novel targeted therapies offer potential additional therapeutic options; however, additional research is required.

(iii) Radiotherapy either single- or multifraction regimes offer symptomatic benefits for bone pain and localised pelvic symptoms.

(iv) Patients with metastatic urothelial cancer demonstrate substantial morbidity that would benefit from close involvement with palliative care services. 


\section{References}

[1] Office of National Statistics, "Cancer registration statistics England 2008," 2010. http://www.statistics.gov.uk.

[2] ICD online. Cancer in Scotland 2010, http://isdscotland.org/ Health-Topics/Cancer/Cancer-Statistics/.

[3] Northern Ireland Cancer Registry Bladder Cancer, 2010, http://www.qub.ac.uk/research-centres/nicr/Data/OnlineStatistics.

[4] Cancer incidence in Wales 2004-2008, 2010, http://www .wales.nhs.uk.

[5] Office of national Statistics, "Mortality Statistics: deaths registered in England and Wales," 2010, http://www.statistics .gov.uk.

[6] M. J. Droller, Bladder Cancer-Current Diagnosis and Treatment, Human Press, 2001.

[7] C. N. Sternberg, P. H. M. de Mulder, J. H. Schornagel et al., "Randomized phase III trial of high-dose-intensity methotrexate, vinblastine, doxorubicin, and cisplatin (MVAC) chemotherapy and recombinant human granulocyte colonystimulating factor versus classic MVAC in advanced urothelial tract tumors: European organization for research and treatment of cancer protocol no. 30924," Journal of Clinical Oncology, vol. 19, no. 10, pp. 2638-2646, 2001.

[8] H. Von der Maase, S. W. Hansen, J. T. Roberts et al., "Gemcitabine and cisplatin versus methotrexate, vinblastine, doxorubicin, and cisplatin in advanced or metastatic bladder cancer: results of a large, randomized, multinational, multicenter, phase III study," Journal of Clinical Oncology, vol. 18, no. 17, pp. 3068-3077, 2000.

[9] T. H. Ecke, H. Gerullis, P. Bartel, S. Koch, and J. Ruttloff, "Palliative chemotherapy with gemcitabine, paclitaxel, and cisplatin as first-line treatment following gemcitabine monotherapy for patients with transitional cell carcinoma of the urothelium," Minerva Urologica e Nefrologica, vol. 61, no. 1, pp. 1-8, 2009.

[10] J. Bellmunt, A. Ribas, N. Eres et al., "Carboplatin-based versus cisplatin-based chemotherapy in the treatment of surgically incurable advanced bladder carcinoma," Cancer, vol. 80, no. 10, pp. 1966-1972, 1997.

[11] L. Dogliotti, G. Cartenì, S. Siena et al., "gemcitabine plus cisplatin versus gemcitabine plus carboplatin as first-line chemotherapy in advanced transitional cell carcinoma of the urothelium: results of a randomized phase 2 trial," European Urology, vol. 52, no. 1, pp. 134-141, 2007.

[12] R. Dreicer, J. Manola, B. J. Roth et al., "Phase III trial of methotrexate, vinblastine, doxorubicin, and cisplatin versus carboplatin and paclitaxel in patients with advanced carcinoma of the urothelium: a trial of the Eastern Cooperative Oncology Group," Cancer, vol. 100, no. 8, pp. 1639-1645, 2004.

[13] J. Carles, E. Esteban, M. Climent et al., "Gemcitabine and oxaliplatin combination: a multicenter phase II trial in unfit patients with locally advanced or metastatic urothelial cancer," Annals of Oncology, vol. 18, no. 8, pp. 1359-1362, 2007.

[14] J. Bellmunt, C. Théodore, T. Demkov et al., "Phase III trial of vinflunine plus best supportive care compared with best supportive care alone after a platinum-containing regimen in patients with advanced transitional cell carcinoma of the urothelial tract," Journal of Clinical Oncology, vol. 27, no. 27, pp. 4454-4461, 2009.

[15] F. Joly, N. Houédé, and S. Noal, "Do patients with advanced urothelial carcinoma benefit from weekly paclitaxel chemotherapy? A GETUG phase II study," Clinical Genitourinary Cancer, vol. 7, no. 2, pp. E28-E33, 2009.

[16] G. Fechner, R. Siener, M. Reimann, L. Kobalz, and P. Albers, "Randomised phase II trial of gemcitabine and paclitaxel second-line chemotherapy in patients with transitional cell carcinoma (AUO Trial AB 20/99)," International Journal of Clinical Practice, vol. 60, no. 1, pp. 27-31, 2006.

[17] C. J. Sweeney, B. J. Roth, F. F. Kabbinavar et al., "Phase II study of pemetrexed for second-line treatment of transitional cell cancer of the urothelium," Journal of Clinical Oncology, vol. 24, no. 21, pp. 3451-3457, 2006.

[18] M. H. A. Hussain, G. R. MacVicar, D. P. Petrylak et al., "Trastuzumab, paclitaxel, carboplatin, and gemcitabine in advanced human epidermal growth factor receptor-2/neupositive urothelial carcinoma: results of a multicenter phase II National Cancer Institute trial," Journal of Clinical Oncology, vol. 25, no. 16, pp. 2218-2224, 2007.

[19] C. Wulfing, J. P. H. Machiels, D. J. Richel et al., "A singlearm, multicenter, open-label phase 2 study of lapatinib as the second-line treatment of patients with locally advanced or metastatic transitional cell carcinoma," Cancer, vol. 115, no. 13, pp. 2881-2890, 2009.

[20] N. M. Hahn, W. M. Stadler, R. Zon et al., "Phase II trial of cisplatin, gemcitabine, and bevacizumab as first-line therapy for metastatic urothelial carcinoma: Hoosier Oncology Group GU 04-75," Journal of Clinical Oncology, vol. 29, no. 12, pp. 1525-1530, 2011.

[21] J. E. Rosenberg, S. Halabi, B. L. Sanford et al., "Phase II study of bortezomib in patients with previously treated advanced urothelial tract transitional cell carcinoma: CALGB 90207," Annals of Oncology, vol. 19, no. 5, pp. 946-950, 2008.

[22] G. Gomez-Abuin, E. Winquist, W. M. Stadler et al., "A phase II study of PS-341 (Bortezomib) in advanced or metastatic urothelial cancer. A trial of the Princess Margaret Hospital and University of Chicago phase II consortia," Investigational New Drugs, vol. 25, no. 2, pp. 181-185, 2007.

[23] Vorinostat in Treating Patients With Locally Recurrent or Metastatic Cancer of the Urothelium, 2010, http://clinicaltrials.gov/ct/show/NCT00363883.

[24] Vorinostat Plus Radiation Therapy in Pancreatic Cancer, 2010, http://www.cancer.gov/search/ViewClinicalTrials.aspx?cdrid= 634674\&version $=$ HealthProfessional\&protocolsearchid $=$ 7865385 .

[25] Vorinostat in Combination With Radiation Therapy and Infusional Fluorouracil (5-FU) in Patients With Locally Advanced Adenocarcinoma of the Pancreas, 2010, http://www. cancer.gov/search/ViewClinicalTrials.aspx?cdrid $=651321 \& v e-$ rsion=HealthProfessional\&protocolsearchid $=7865385$.

[26] Phase I Study of Chemoradiotherapy Comprising Capecitabine and Vorinostat in Patients With Nonmetastatic Pancreatic Cancer, 2010, http://www.cancer.gov/search/ViewClinicalTrials.aspx?cdrid $=655043 \&$ version $=$ HealthProfessional\&protocolsearchid $=7865385$.

[27] A. H. Ree, S. Dueland, S. Folkvord et al., "Vorinostat, a histone deacetylase inhibitor, combined with pelvic palliative radiotherapy for gastrointestinal carcinoma: the Pelvic Radiation and Vorinostat (PRAVO) phase 1 study," The Lancet Oncology, vol. 11, no. 5, pp. 459-464, 2010.

[28] M. Peyromaure, F. Scotté, D. Amsellem-Ouazana, A. Vieillefond, S. Oudard, and P. Beuzeboc, "Trastuzumab (Herceptin $^{(T M)}$ ) in metastatic transitional cell carcinoma of the urinary tract: report on six patients," European Urology, vol. 48 , no. 5, pp. 771-775, 2005. 
[29] P. Beuzeboc, E. Banu, E. Voog et al., "Trastuzumab (T) combined with standard chemotherapy in HER+ metastatic bladder cancer (BC) patients: interim safety results of a prospective randomized phase II study," Journal of Clinical Oncology, vol. 25, article 15565, no. 18S, 2007.

[30] D. Cameron, "Lapatinib plus capecitabine in patients with HER2-positive advanced breast cancer," Clinical Advances in Hematology and Oncology, vol. 5, no. 6, pp. 456-458, 2007.

[31] B. H. Bochner, R. J. Cote, N. Weidner et al., "Angiogenesis in bladder cancer: relationship between microvessel density and tumor prognosis," Journal of the National Cancer Institute, vol. 87, no. 21, pp. 1603-1612, 1995.

[32] G. M. Duchesne, J. J. Bolger, G. O. Griffiths et al., "A randomized trial of hypofractionated schedules of palliative radiotherapy in the management of bladder carcinoma: results of medical research council trial BA09," International Journal of Radiation Oncology, vol. 47, no. 2, pp. 379-388, 2000.

[33] D. B. McLaren, D. Morrey, and M. D. Mason, "Hypofractionated radiotherapy for muscle invasive bladder cancer in the elderly," Radiotherapy \& Oncology, vol. 43, no. 2, pp. 171-174, 1997.

[34] J. R. Yarnold, " 8 Gy single fraction radiotherapy for the treatment of metastatic skeletal pain: randomised comparison with a multifraction schedule over 12 months of patient follow-up. Bone Pain Trial Working Party," Radiotherapy \& Oncology, vol. 52, no. 2, pp. 111-121, 1999.

[35] E. Steenland, J. W. Leer, H. van Houwelingen et al., "The effect of a single fraction compared to multiple fractions on painful bone metastases: a global analysis of the Dutch Bone Metastasis Study," Radiotherapy \& Oncology, vol. 52, no. 2, pp. 101-109, 1999.

[36] W. F. Harstell, C. B. Scott, D. W. Bruner et al., "Randomized trial of short- versus long-course radiotherapy for palliation of painful bone metastases," Journal of the National Cancer Institute, vol. 97, no. 11, pp. 798-804, 2005.

[37] S. K. Sarkar, S. Sarkar, B. Pahari, and D. Majumdar, "Multiple and single fraction palliative radiotherapy in bone secondaries - A prospective study," Indian Journal of Radiology and Imaging, vol. 12, no. 2, pp. 281-284, 2002.

[38] W. M. Sze, M. Shelley, I. Held et al., "Palliation of metastatic bone pain: single fraction versus multifraction radiotherapy," Cochrane Database of Systematic Reviews, no. 2, 2004.

[39] G. N. Hortobagyi, R. L. Theriault, A. Lipton et al., "Longterm prevention of skeletal complications of metastatic breast cancer with pamidronate. Protocol 19 Aredia Breast Cancer Study Group," Journal of Clinical Oncology, vol. 16, no. 6, pp. 2038-2044, 1998.

[40] N. Norio Kohno, K. Aogi, H. Minami et al., "Zoledronic acid significantly reduces skeletal complications compared with placebo in Japanese women with bone metastases from breast cancer: a randomized, placebo-controlled trial," Journal of Clinical Oncology, vol. 23, no. 15, pp. 3314-3321, 2005.

[41] F. Saad, D. M. Gleason, R. Murray et al., "Long-term efficacy of zoledronic acid for the prevention of skeletal complications in patients with metastatic hormone-refractory prostate cancer," Journal of the National Cancer Institute, vol. 96, no. 11, pp. 879-882, 2004.

[42] M. S. Zaghloul, R. Boutrus, H. El-Hossieny, Y. A. Kader, I. ElAttar, and M. Nazmy, "A prospective, randomized, placebocontrolled trial of zoledronic acid in bony metastatic bladder cancer," International Journal of Clinical Oncology, vol. 15, no. 4, pp. 382-389, 2010.
[43] J. R. Berenson, A. Lichtenstein, L. Porter et al., "Longterm pamidronate treatment of advanced multiple myeloma patients reduces skeletal events. Myeloma Aredia Study Group," Journal of Clinical Oncology, vol. 16, no. 2, pp. 593602, 1998.

[44] Summary of Product Characteristics. Zometa $4 \mathrm{mg} / 5 \mathrm{ml}$ Concentrate for Solution for Infusion, 2010, http://www.medicines.org.uk.

[45] Guidance on Cancer Services. Improving Outcomes in Urological Cancers. The Manual. National Institute of Clinical Excellence, 2002.

[46] R. D. Brierly and T. S. O'Brien, "The importance of palliative care in urology," Urologia Internationalis, vol. 80, no. 1, pp. 13$18,2008$. 


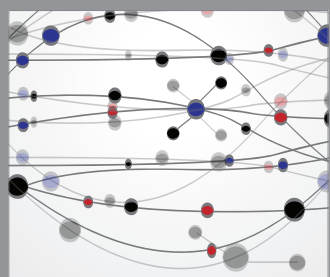

The Scientific World Journal
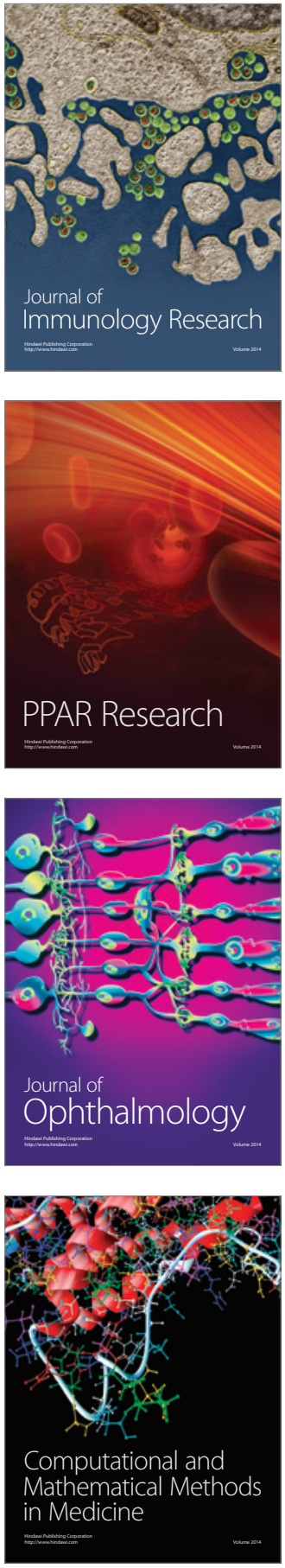

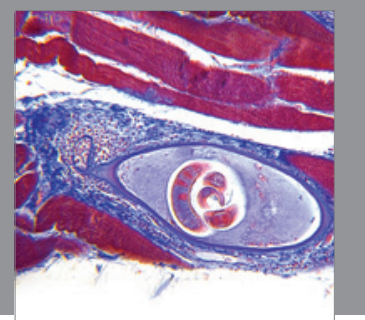

Gastroenterology

Research and Practice
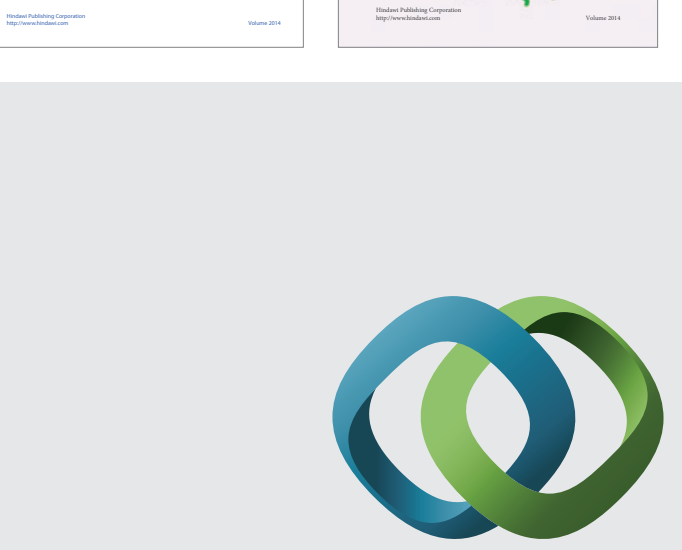

\section{Hindawi}

Submit your manuscripts at

http://www.hindawi.com
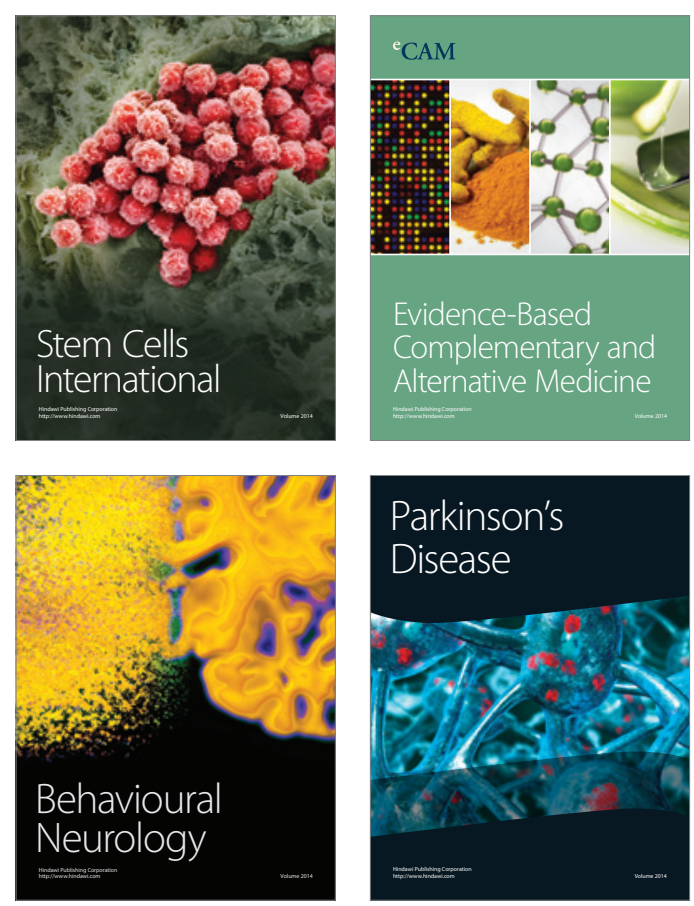

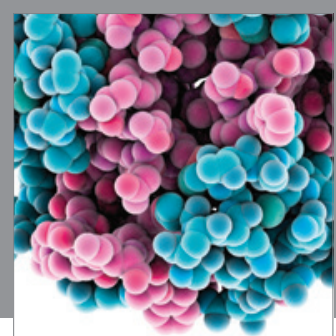

Journal of
Diabetes Research

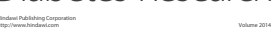

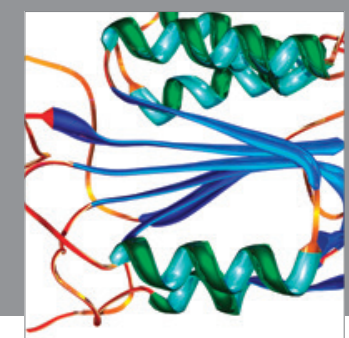

Disease Markers
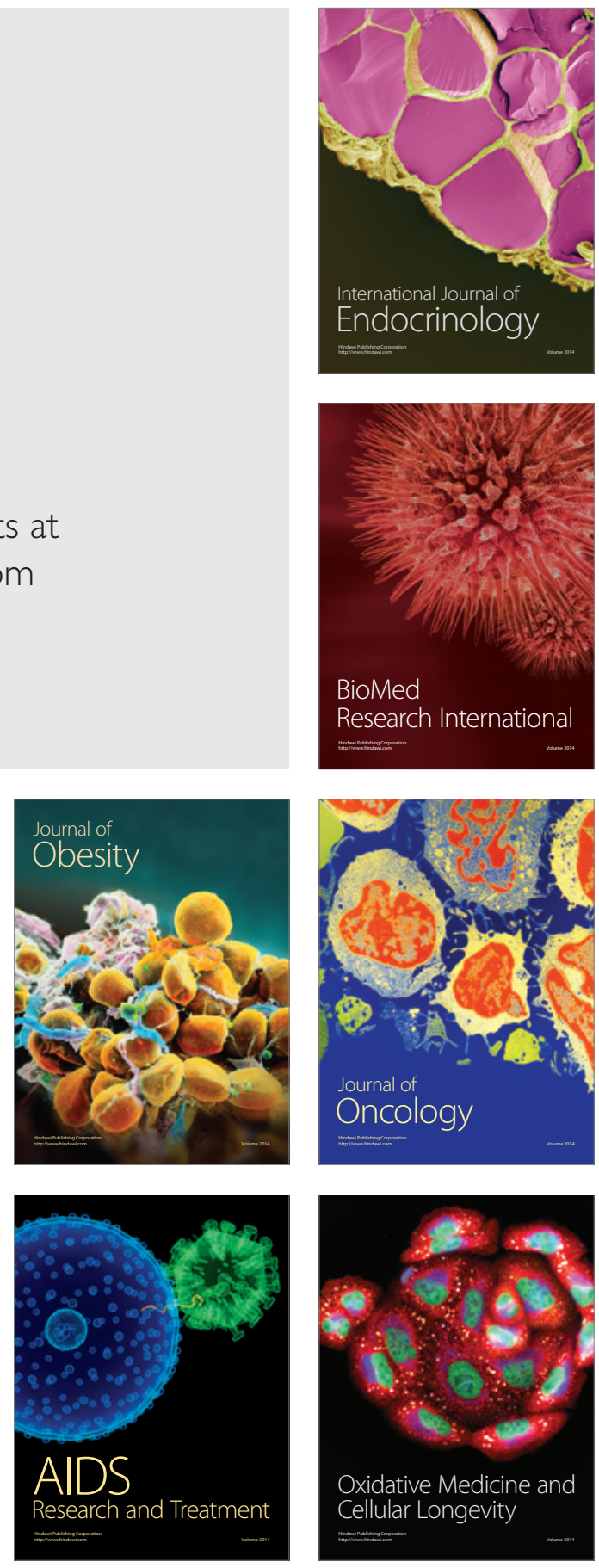